\title{
Squeezed light in optomechanical systems
}

Harris, G. I.; Taylor, M. A.; Hoff, Ulrich Busk; Janousek, J.; Daria, V.; Knittel, J.; Kerdoncuff, Hugo; Hage, B.; Andersen, Ulrik Lund; Bachor, H.-A.

Total number of authors:

11

Published in:

Proceedings of Frontiers in Optics (FiO)

Publication date:

2012

Document Version

Publisher's PDF, also known as Version of record

Link back to DTU Orbit

Citation (APA):

Harris, G. I., Taylor, M. A., Hoff, U. B., Janousek, J., Daria, V., Knittel, J., Kerdoncuff, H., Hage, B., Andersen, U. L., Bachor, H-A., \& Bowen, W. P. (2012). Squeezed light in optomechanical systems. In Proceedings of Frontiers in Optics (FiO) (pp. FW5C.4). Optical Society of America.

\section{General rights}

Copyright and moral rights for the publications made accessible in the public portal are retained by the authors and/or other copyright owners and it is a condition of accessing publications that users recognise and abide by the legal requirements associated with these rights.

- Users may download and print one copy of any publication from the public portal for the purpose of private study or research.

- You may not further distribute the material or use it for any profit-making activity or commercial gain

- You may freely distribute the URL identifying the publication in the public portal 


\title{
Squeezed light in optomechanical systems
}

\author{
G. I. Harris ${ }^{1}$, M. A. Taylor ${ }^{1}$, U. Busk-Hoff ${ }^{3}$, J. Janousek ${ }^{2}$, V. Daria ${ }^{2}$, J. Knittel ${ }^{1}$, H. Kerdoncuff ${ }^{3}$, B. Hage ${ }^{2}$, U. \\ L. Andersen ${ }^{3}$, H.-A. Bachor ${ }^{2}$, and W. P. Bowen ${ }^{1}$ \\ ${ }^{1}$ Centre of Excellence in Engineered Quantum Systems, University of Queensland, St. Lucia, Queensland 4072, Australia \\ ${ }^{2}$ Department of Quantum Science, Australian National University, Canberra, ACT 0200, Australia \\ ${ }^{3}$ Department of Physics, Technical University of Denmark, Building 309, 2800 Lyngby, Denmark \\ Presenting author e-mail address: glen.harris@uqconnect.edu.au
}

\begin{abstract}
Squeezed light enhanced optomechanical measurements are demonstrated in both intracavity and biological contexts, with respective enhancements of 1.0 and $2.7 \mathrm{~dB}$. Quantum enhanced microrheology of the cytoplasm of a yeast cell is thereby realized.

OCIS codes: (270.0270) Quantum optics, (230.4685) Optical microelectromechanical devices, (120.4880) Optomechanics.
\end{abstract}

\section{Introduction}

The quantum nature of light places a fundamental limit on the sensitivity of optical measurements. In circumstances with constrained optical power, this limit may only be surpassed using non-classical resources. Despite the promise of non-classical resources, to date the sole example of a real application is in interferometric gravity wave detection where the optical power is constrained due to absorptive heating[1]. Much broader and widely discussed applications are possible in the areas of quantum optomechanics, where ultimately a non-classical light field interacts with a non-classical mechanical oscillator, and biological sensing[2], where low light levels are often required to avoid damaging the specimen. Here we report two distinct experiments which use squeezed light to, for the first time, achieve quantum enhanced sensitivity in biological and cavity optomechanical contexts, with 2.7 and $1.0 \mathrm{~dB}$ of enhancement achieved, respectively. The biological experiments, particularly, allow quantum enhanced microrheology to be performed on the cytoplasm of a Saccharomyces cerevisiae yeast cell, revealing subdiffusive motion. This provides a pathway towards microrheology of cell mechanics and the cytoskeleton at high frequencies, where motion amplitudes are beneath the sensitivity of current technology.

\section{Microcavity experiment}

As shown in Fig. 1, phase squeezed light was injected, on resonance, into a silicon chip based microtoroidal resonator. Microtoroidal resonators exhibit high quality mechanical modes, with an example shown in Fig. 1 (right), which have been cooled close to their quantum ground state[3]. The output field from the microtoroid was interfered with a bright local oscillator field on a 50/50 beam splitter. The non-classical photon correlations inherent to phase squeezing allowed the mechanical motion of the microtoroid to be transduced with $1 \mathrm{~dB}$ higher sensitivity than the quantum noise limit introduced by quantization of light.

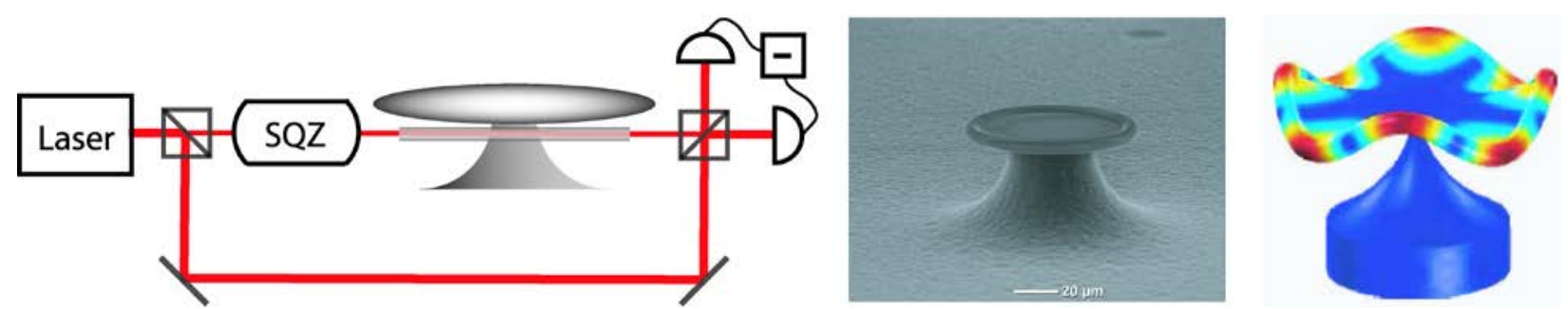

Fig. 1. Left: schematic of cavity optomechanical squeezing experiment. SQZ: squeezer. Center right: SEM image of microtoroid. Far right: microtoroid mechanical mode.

\section{Optical tweezers experiment}

The apparatus used to demonstrated sub-quantum noise limited sensitivity in a biological context is shown in Fig. 1. A specimen was suspended in water within a sample chamber formed by two microscope coverslips, and trapped with a dual beam optical trap at $1064 \mathrm{~nm}$. An orthogonally polarized amplitude squeezed local oscillator field was spatially engineered using a phase plate for maximum sensitivity to motion of the specimen (see vertical panels on the left of Fig. 2) and injected into an optical tweezers. A further probe laser field, coherent with the squeezed local oscillator field, was injected transversely to the optical axis of the tweezers. Scattering of this field interfered with 
the squeezed field, encoding position information about the specimen which could be retrieved via direct detection. Apart from the deleterious effects of optical losses and spatial distortion in the objectives and specimen, a major technical challenge was to minimize exposure to noise sources at the sub $\sim \mathrm{kHz}$ frequencies relevant to biological motion. This was achieved by stroboscopically pulsing the probe field at $3.522 \sim \mathrm{MHz}$, which had the effect of mixing up the motion into the region of strongest squeezing. This technique should be broadly applicable to squeezed light enhanced sensors.

Motion sensitivity surpassing the quantum noise limit by up to $2.7 \sim \mathrm{dB}$ and 2.4 $\mathrm{dB}$ was achieved, respectively, for trapped silica beads and lipid granules within a yeast cell; in both cases allowing the mean squared displacement of the specimen to be more accurately measured than is possible with classical light. The mean squared displacement measurements for yeast with (orange) and without (blue) squeezing are shown in Fig. 2 (right), clearing demonstrating the superiority of measurements with squeezed light. The lipid granule exhibits sub-diffusive motion as it interacts with the cells cytoplasm, consistent with recent classical measurements with a different yeast strain[4].
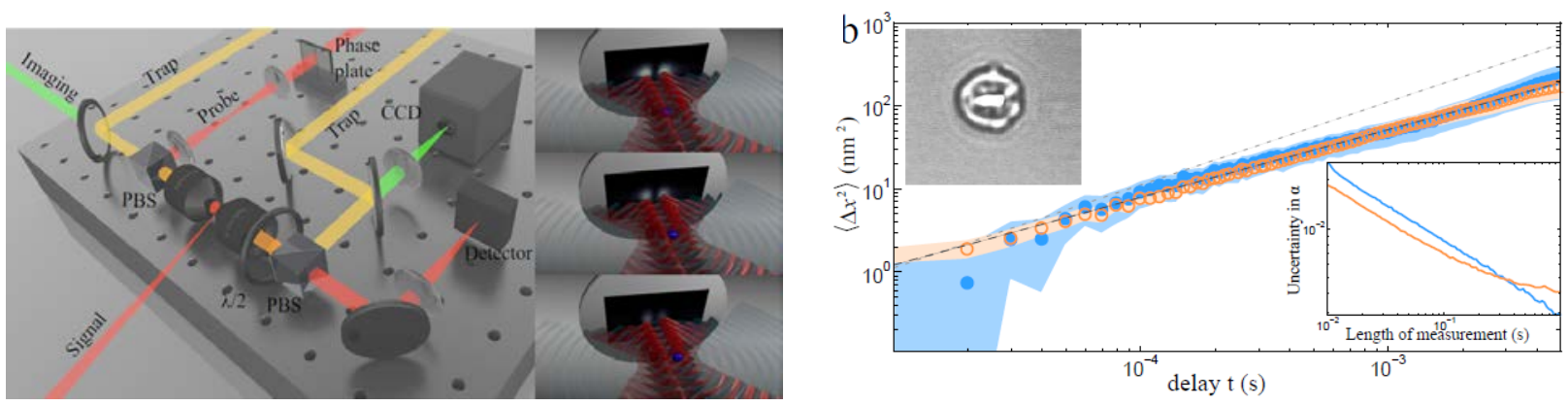

Fig.2. Left: experimental schematic, vertical panels show the probe (red) and scattered (blue) fields, illustrating dependence of detected intensity on particle position. Right: mean squared displacement measurements on lipid particle within a yeast cell. Orange and blue respectively indicate measurement results and uncertainty with and without squeezed light. Dashed line: expected mean squared displacement for diffusive motion.

\section{Conclusion}

We have demonstrated optomechanical transduction sensitivity surpassing the quantum noise limit in both cavity optomechanical systems, and optical tweezers. These experiments represent enabling steps towards new practical sensors, including force measurement beyond the standard quantum limit, measurements of the non-Einsteinian Brownian motion[5], and low light level high bandwidth sensing of biological dynamics within living cells[6].

\section{Acknowledgements}

This research was funded by the ARC Centre of Excellence CE110001013 and Discovery Project DP0987146. Device fabrication was undertaken within the Queensland Node of the Australian Nanofabrication Facility.

\section{References}

[1] H. Vahlbruch et al. Class. Quantum Grav. 27084027 (2010)

[2] N. Treps et al. Phys. Rev. Lett. 88, 203601 (2002)

[3] E. Verhagen, S. Deléglise, S. Weis, A. Schliesser, T. J. Kippenberg, Nature 48263 (2012).

[4] I. M. Tolic-Norrelykke et al. Phys. Rev. Lett. 93, 078102 (2004).

[5] R. Huang, I. Chavez, K. M. Taute, B. Lukic, S. Jeney, M. G. Raizen, and E.-L. Florin, Nature Phys. 7576580 (2011).

[6] S. Yamada, D. Wirtz, and S. C. Kuo, Biophys. J. 78, 17361747 (2000). 